\section{Abstractions}

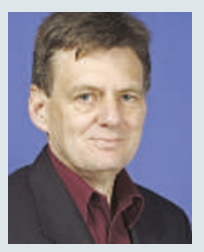

\section{LAST AUTHOR}

The malaria parasite can evade our immune system by altering the way it looks during the infection. One way it does this is by frequently changing the version of a protein, PfEMP1, it leaves on the surface of red blood cells it infects. Figuring out the molecular mechanism behind this process, called antigenic variation, could offer clues on how to design effective vaccines or drugs. Sixty different members of a gene family called var each code for a different version of PfEMP1, but only one of these genes is turned on at a time. Alan Cowman of the Walter and Eliza Hall Institute of Medical Research in Parkville, Australia, and his colleagues are investigating how the parasite manages to keep all but one gene silent. On page 1004, they report that an active 'promoter' - a regulatory stretch of DNA adjacent to the genes - is sufficient to inactivate all but one of the var genes. Cowman spoke to Nature about the implications of his results.

How can this finding be applied to the development of more effective malaria drugs or vaccines?

It increases our understanding of the basic biology of the parasite. Also, understanding how the parasite controls the expression of the var gene family provides the possibility of developing compounds that inhibit the process or alternatively allow the simultaneous expression of all of the PfEMP1 proteins. While these would be useful experimental tools, they would also allow us to ask the question: would these compounds be useful drugs if they do not kill the parasite but make it more open to immune attack?

How has the malaria parasite managed to get so good at evading immune responses? There is enormous selective pressure placed on it by the host immune system. It has come up with a number of mechanisms to evade immune responses at different stages of its life cycle. It also has numbers on its side. There can be up to $10^{12}$ parasites in a single infection and this provides enormous potential for variation.

Can we develop an effective malaria vaccine? Humans can develop good immunity to malaria, which suggests that it is possible, but it requires an understanding of the mechanisms and the parasite molecules involved. I think with a continued and increasing investment in both basic and translational research over a long period of time and with effort and perseverance, it should be possible.

\section{What's the next step in this research?} We want to identify the proteins directly involved in silencing and activating the var gene family and therefore the molecular framework that controls antigenic variation.

\section{MAKING THE PAPER}

\section{Onur Hosten}

A quantum computer can solve
problems without running a program.

When we look at matter on the scale of atoms and electrons, we see counterintuitive behaviour. Quantum particles, for example, can exist in two states at once, a phenomenon called superposition. Famously, Erwin Schrödinger used the image of a cat that is both dead and alive to illustrate this dissonance between what quantum theory tells us and what we observe in our everyday experience. But even for quantum physicists, the notion of getting information from a computer without turning it on seems odd. "Everyone thinks it's weird. It is unintuitive," says Onur Hosten, a graduate student at the University of Illinois at UrbanaChampaign.

Hosten's work demonstrating counterfactual computation (CFC) - the process by which a quantum computer's solution to a computation may be learnt without actually running the computer - appears on page 949 of this issue. Strange as it is, CFC is not a new concept. "It was shown to be theoretically possible in 1998, but thought to be no better than random guessing," explains Hosten.

When Hosten started working on the project in 2004, at the suggestion of his supervisor, Paul Kwiat, few people were investigating the phenomenon. "It was thought odd and of no practical utility," says Hosten. But what began as an exercise in theory led to interesting and potentially useful results. He demonstrated that CFC was physically possible. "I then came up with the idea of beating the random-guessing limit," he adds. Hosten showed that CFC could be used to infer the outcome of a computation with a higher probability of getting the right answer than random guessing. He then spent the following year thinking about possible applications for his finding. "We found that with CFC you can avoid some errors that are happening in the computer," he

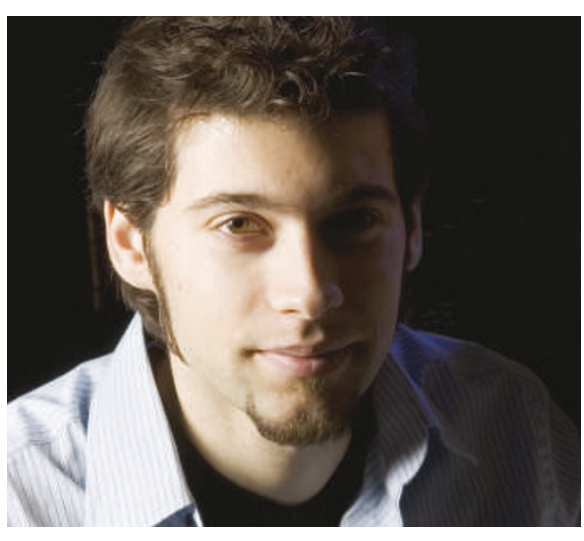

explains, a discovery that could lead to methods for making quantum computing more robust and feasible.

The key to Hosten's success was finding a way to superpose the states 'running' and 'not running' in a quantum computer. He used an optical approach: a single photon could travel through the computer (representing the computer running) or an empty path (computer not running). Because in quantum physics the photon, like Schrödinger's cat, can simultaneously exist in different places or states, this allowed Hosten to superpose the computer's on and off states and infer an answer to a computation without running the computer. "Although you could get the result without running the computer, you still need the computer to be there, and you need to give it the same time to function as if it were running. Otherwise you cannot superpose the two histories to get the result," says Hosten. Sounds complicated? It is. "I understand the process in terms of the mathematics and physics involved, but if I get into very deep philosophical questions, I will fail," Hosten adds.

While others in the field ponder his study, Hosten has moved to the next challenge, quantum non-demolition measurements, or the ability to detect a photon's presence without destroying it in the process. Once again, the process has an established theory, but no one has put it to the test yet. "That's what I do," says Hosten.

\title{
QUANTIFIED FINLAND
}

\section{A numerical perspective on Nature authors.}

Finland's cold climate, plus its perceived isolation, make recruiting hard work for Jussi Taipale - a principal investigator in the molecular and cancer biology programme at the University of Helsinki. Nevertheless, he has built a talented team in his growth-control lab. Taipale says the excitement of the science is a pull, but so too is the freedom he offers team members to pursue their own goals. Taipale doesn't believe in micro-managing the lab's daily activities. Rather, he looks for people who can work independently and creatively within the group's broad research plan.

His strategy seems to be paying off. Two of his postdocs, Mikael Björklund and Minna Taipale, have led the lab's efforts in automated data analysis, allowing them to identify the genes controlling cell division and cell size (see page 1009).
395 is the number of doctoral degrees awarded by the University of Helsinki in 2004

8 is the number of postdocs and students working in Taipale's team.

$\mathbf{6}$ is the number of papers published by Nature over the past year with contributing authors working in Finland.

\section{$\mathbf{8}, \mathbf{5 5 0}$ is the total number of} citations made, at the time of writing, to Taipale's three previous Nature publications. 